\title{
PAPEL DE LA CONFIANZA en las relaciones entre organizaciones
}

\section{iresUMEN}

El objetivo del presente documento es analizar las definiciones y la importancia de la confianza para crear relaciones de cooperación entre organizaciones de naturaleza distinta. Se hace referencia a los modelos de organización dominantes para acercarnos a los nuevos mecanismos de regulación social propuestos por la sociología para tratar la organización, debido a que en el contexto actual las nuevas concepciones de la organización del trabajo han promovido en las organizaciones formas distintas de competir en el mercado.

Procesos de cambio que obligan a las organizaciones a construir una nueva identidad y a renovar los contratos psicológicos con sus integrantes. La noción de confianza ha sido llevada del ámbito social al organizacional con la finalidad de lograr acuerdos entre organizaciones $y$ al mismo tiempo lograr el compromiso de los integrantes de tales entidades. Para que estas puedan funcionar bajo este marco de transformaciones requieren de relaciones de colaboración regidas por la confianza; relaciones que se dan en los niveles individual, dentro de la organización, entre organizaciones e institucionalmente. Los diferentes niveles de confianza están presentes en las relaciones que entablan los individuos, tanto dentro de la organización como en su entorno. Algunas de las reflexiones en torno a esta problemática nos llevan a pensar que el papel de la confianza es dinámico e incide en la transferencia y el intercambio de conocimientos, técnicas y procedimientos científicos, tecnologías, productos, capitales, entre otros. Además, las relaciones fundadas en la confianza requieren de la suspensión del propio interés, en beneficio de una orientación colectiva (Mangematin y Thuderoz, 2003). Sin embargo, también se reconoce que con todo y la posibilidad que el hombre tiene de deliberar y de generar confianza, es evidente que entre los hombres prevalecen tanto la confianza calculada como los deseos de altruismo para entrar en la confianza interpersonal.

\section{alabras clave}

Confianza, identidad, relaciones organizacionales, mecanismo de regulación social y modernización.

\author{
Por: \\ Irene Juana Guillén Mondragón \\ Laura Patricia Peñalva Rosales ${ }^{I}$
}

$\mathbf{E}$ desarrollo económico mundial obliga a todas las naciones a entrar en el juego de la globalización, en el cual se hacen más evidentes las desigualdades entre las economías desarrolladas y las menos favorecidas, como la nuestra. La complejidad y las transformaciones en el esquema de competencia económica, así como la creciente crisis social-con problemas de fragmentación, pobreza, desempleo, violencia y contaminación por mencionar sólo algunos-, exigen a las organizaciones el replanteamiento de sus esquemas de trabajo, tanto en sus procesos y tecnologías como en sus formas de regulación social.

La racionalidad, que subyace en las dinámicas de cambio que las organizaciones se ven obligadas a realizar, sólo ha tomado en cuenta

\footnotetext{
${ }^{1}$ Irene Juana Guillén Mondragón. Profesora-Investigadora del Departamento de Economía de la Universidad Autónoma Metropolitana, Unidad-Iztapalapa. Miembro del Área de Investigación «Planeación Estratégica de las Empresas» y del Cuerpo Académico «Estrategia Empresarial Mexicana»; Laura Patricia Peñalva Rosales. Profesora -Investigadora de la Universidad autónoma Metropolitana, Unidad-Xochimilco. Miembro del Área de Investigación «Estrategia y Gestión de las Organizaciones Mexicanas» y del Cuerpo Académico «Laboratorio de Ingeniería en Management Socioeconómico».
} 
la eficiencia y la eficacia como valores fundamentales para mantenerse dentro del orden económico, sin reflexionar que tales acciones inciden profundamente en el individuo y en la sociedad.

En el caso de México, las acciones políticas de los gobiernos neoliberales, en su deseo por sumarse al primer mundo, han llevado a poner en práctica estrategias de modernización y de cambio que obligan a las organizaciones en general ${ }^{1}$ a adoptar modelos gerenciales para, según ellos, aprovechar oportunidades de mercado (mercados globales), pero que a la vez las conduce a enfrentar mayor competencia y nuevos retos.

El proceso de modernización constituye uno de los desafíos más importantes para la mayoría de las empresas en nuestro país. Ante esta situación, es imperativo ampliar los horizontes hasta límites que hasta hace pocos años eran impensados. Por otra parte, el éxito o fracaso de la industria mexicana repercute en los ámbitos sociales y económicos, pues este es un sector que contribuye al mejoramiento del nivel de vida de la colectividad, tal como ocurre con las pequeñas y medianas empresas (PyMES), que contribuyen con el mayor porcentaje de empleo a escala nacional, al tiempo que generan flujos de utilidad económica y social para quienes la forman (Camargo, 2001). En este tipo de organizaciones, tanto los dirigentes como los trabajadores aportan esfuerzos al desarrollo social, pues para ellos es importante que sus organizaciones sobrevivan, con lo cual, en la medida de lo posible, se garantiza el bienestar familiar y colectivo.

\section{ABstract}

The objective of this paper is to analyze the importance and the definitions of the trust for creating relations of cooperation between organizations of different nature. One refers about the organization dominant models to approach to social regulation new means proposed by sociology to deal the with the organization; due to that in actual context the new conceptions of work organization have promoted in the organization different ways of competition in the market.

Changing processes which force the organizations to enact new identities and to update the psychological contracts with their members. The notion of trust has been taken from the social sphere to the organizational one with the purpose of achieving agreements between organizations and at the same time accomplishing the commitment of the coworkers from such entities. In order to keep the organizations under this frame of transformations they need collaboration relationships governed by trust. There are three kinds or relationships that exist in the individual level, inside the organization, among the organizations and institutionally, The different levels of trust are present in the relations that the individuals being, so much inside the organization like in its environment.

Some of the reflections about this problematic lead us to think that the role of trust is dynamical and influences in the knowledge transference and/or interchange, technical methods, scientific knowledge, technology, products, capital, and so on. Moreover, relationship based in trust demand pulling off the own interest in benefit of a collective orientation (Mangematin y Thuderoz, 2003).

However, it is also recognized that although the human being has the right to deliberate (o has his own free will) and to enact trust, is evident that between human beings still prevail so much both the calculated trust and the wishes of selflessness of altruism for getting into interpersonal trust.

\section{KEY WORDS}

Trust, identity, relationships, organizations, means of social regulation and modernization.

\footnotetext{
1 En México, el 99.7\% de empresas corresponde al estrato de micro, pequeñas y medianas empresas (www.economia.gob.mx).
} 
En el caso de las empresas mexicanas y en el contexto de la actual modernización impuesta por las políticas gubernamentales, la confianza juega un papel crucial en tanto influye en el orden cultural y en la identidad. Se requiere de una nueva forma de regulación social (Sainsaulieu, 1997) en la que la identidad y la generación de confianza jueguen un papel fundamental para que los trabajadores cooperen y se comprometan con los objetivos de sus organizaciones en la búsqueda de un mejor desarrollo social.

Este es el caso, por ejemplo de los modelos de calidad que se transfieren a las pequeñas empresas en el sector manufacturero de autopartes, que, por su tendencia hacia la innovación, pueden generar varias lógicas de acción colectiva. Según Alter (citado por Sainsaulieu, 1997), esas lógicas van desde las que desean conservar el statu quo, hasta las que modifican costumbres y relaciones colectivas, sin olvidar las lógicas de acción de los excluidos, las cuales de alguna manera afectan a la vez el desempeño de la organización y de la sociedad. La experiencia colectiva de la innovación ayuda a formar y transformar la cultura de la organización y a marcar el posicionamiento y los recursos de negociación entre los actores.

Las transformaciones que sufren las organizaciones en sus procesos de cambio conllevan la necesidad de construir nuevas identidades. La construcción de la identidad colectiva es dinámica y oscilante, sobre todo si se refiere a la identidad de la organización, cuyo fin es moldear a sus miembros para poder predecir sus comportamientos. En este sentido, la identidad se convierte en un medio de regulación social (Sainsaulieu, 1997), el cual según Alvesson (2002) constituye un medio de control cultural articulado y legitimado por los propios miembros de la organización.
Esto no quiere decir que no haya tensión entre el seductor discurso administrativo y la identidad individual de cada actor dentro de la organización. Digamos, entonces, que la identidad de una organización implica, por un lado, la aceptación negociada de los intereses individuales y los del grupo y, por otro, genera incertidumbre dada la disyuntiva a la que se enfrentan los individuos en la elección de valores y significados que deberán compartirse, así como las creencias, las ideas y los símbolos de identificación de la comunidad.

Para Sainsaulieu (1997), la organización es un lugar donde el actor es a la vez individuo, pues se reconoce no sólo en su dimensión de fuerza de trabajo sino también en su dimensión individual y colectiva, a través de su identidad, entendida ésta como un proceso cognitivo en el que se da una definición social que diferencia, que da valor y originalidad, y que permite a los sujetos ser diferentes y obtener el reconocimiento de los otros.

Asimismo, será mediante la generación de confianza como se logre contender con la incertidumbre y como se establezca el diálogo necesario para reconocer valores y compartir significados.

Más adelante, en este documento se presenta la noción de confianza y el lugar que ocupa como un elemento de los nuevos mecanismos de regulación social; también se plantean las diferentes concepciones que sobre este concepto ofrecen varios investigadores para tratar de aclarar su papel en las relaciones sociales y en los procesos de transferencia. Asimismo, se plantean las formas de identidad a que conduce la modernización por medio de la transferencia de nuevas formas de organización del trabajo. Finalmente, en un breve apartado, se esbozan algunas reflexiones preliminares. 


\section{LOS NUEVOS MECANISMOS DE REGULACIÓN SOCIAL EN LA ORGANIZACIÓN}

Con la globalización se ha generado un contexto altamente incierto y de rápidas transformaciones, en el cual se presenta una tendencia isomórfica institucional, que, según Heydebrand (1989), conduce a nuevas formas de organización que emergen como respuesta a los cambios tecnológicos y socioeconómicos. El nuevo orden mundial presenta dinámicas distintas en las que se reconoce una pluralidad de racionalidades (de género, de etnicidad, de cultura, entre otras), las cuales coexisten con la racionalidad instrumental que sólo toma nuevos perfiles.

La globalización y la dinámica de cambios que genera trajeron consigo una forma de organización diferente a la moderna, que se hizo evidente desde los años sesenta con el éxito japonés, y que demostró su fortaleza con la extensa producción de bienes de alta calidad y bajo costo, los cuales llegaron incluso hasta el mercado norteamericano y desplazaron sus productos nacionales. El enfoque de las organizaciones japonesas centradas en el cliente demostró que la racionalidad instrumental no era la única lógica de la acción y que aspectos como la cultura, representaban un sustento diferente para lograr un alto desempeño; de ahí que se pasase de un paradigma cuantitativo a uno cualitativo para explicar las diferentes realidades locales y organizativas de las empresas.
La organización flexible, a diferencia de la burocrática, demanda fuerza de trabajo más educada, con una gran diversidad de conocimientos y competencias, es decir, polivalente, pues por tratarse de una estructura planaflexible- propicia la toma de decisiones descentralizada; en ese proceso se delega a los equipos de trabajo la posibilidad de participar en ella con la finalidad de alcanzar la mejora continua; es decir, se les da poder (empowerment).

La dinámica del cambio actual y el tránsito de un modo de organización rígido a otro flexible requieren reformular los valores en las relaciones de trabajo, dado que ahora la organización del trabajo y gobernabilidad ${ }^{1}$ de la organización son derechos compartidos entre todos sus miembros.

La referencia a los modelos de organización dominantes permite acercarnos a nuevas propuestas sociológicas para tratar la organización. Las nuevas condiciones contextuales, con sus fabulosos cambios tecnológicos y sus nuevas formas de organización del trabajo, obligan a replantearse la concepción de la organización, considerándola como algo más que sólo un arreglo de individuos que comparten intereses o como una cadena de clientes y proveedores cuyos intercambios son siempre igualitarios y cuya lógica es la de relaciones mercantiles.

\footnotetext{
1 En la dinámica actual el concepto de gobernabilidad va acompañado del de gobernanza; para Oriol (2003), la gobernabilidad es la capacidad de un sistema sociopolítico para gobernarse asimismo en el contexto de otros sistemas más amplios de los que forma parte. La gobernanza se da a través de los procesos de interacción entre actores estratégicos. Tales patrones de interacción se fundamentan en las reglas formales e informales que restringen el comportamiento de los actores. En esas interacciones los actores cumplen una doble función; la de solucionar dilemas distributivos y la de corregir problemas de información bajo contextos diferenciados.
} 
Actualmente, el tradicional punto de vista económico sobre el funcionamiento de las organizaciones se complementa con una visión más humanista que matiza la concepción de un mundo regido por el interés personal (Thuderoz, et al., 1999), y por el dominio de la noción de una dirección científica centrada en la mediación de relaciones de producción fundadas en la desconfianza (hacia el asalariado, frente a la desconfianza de éste hacia la organización), y se transita hacia otro en el cual la recomposición de las relaciones de intercambio integra la noción de confianza, en la cual la fe, la creencia y la proximidad son elementos clave que se comparten en las relaciones de trabajo a través de la palabra (Faÿ, 2004 ), para disminuir la incertidumbre ${ }^{1}$.

Sainsaulieu (1997:275) propone concebir a la organización como «una verdadera sociedad de actores comprometidos en un mismo esfuerzo colectivo de producción al encuentro de una triple exigencia: la de la socialización para el trabajo, las estrategias de poder, y las lógicas de acción colectiva...»; en la que se confiere importancia tanto a la racionalidad económica como a la social, es decir, llegar a lo que algunos autores llaman una organización sustentable. Al respecto, Aktouf (1998:302) opina que «toda actividad económica debe inscribirse y subordinarse a un orden y una finalidad social y ecológica que la preserven de grandes desequilibrios y rupturas».

En suma, Aktouf propone buscar una administración más humana y menos impersonal, que contemple no sólo objetivos económicos sino también un proyecto social que justifique su actividad empresarial lucrativa; un proceso administrativo que tome en cuenta a los empleados como actores y no como factores de la producción; a quienes se les delegue autoridad, se les dé confianza y con quienes se comparta valores.

Por otro lado, Wisner (1992) señala que en la organización los individuos reformulan constantemente su sistema social y cultural y, por lo mismo, su identidad; de tal manera que se puede pensar los entramados sociales como redes de relaciones sociales en las cuales se genera un sentido de pertenencia entre los individuos, que a su vez desarrolla lazos de solidaridad y de confianza.

El contexto actual y las nuevas concepciones de la organización y del trabajo han promovido en las organizaciones formas distintas de competir en el mercado. Para que ellas puedan funcionar bajo este marco de transformaciones requieren de relaciones de colaboración regidas por la confianza; relaciones que se dan en los niveles individual, dentro de la organización, entre organizaciones e institucionalmente. En todos esos niveles, la confianza involucra enlaces específicos, en donde hay la suficiente probabilidad de que tanto las personas o las organizaciones con quienes se da la relación, desempeñen una acción benéfica, 0 al menos no perjudicial, para que su contraparte desee considerar comprometerse en alguna forma de cooperación en el futuro (Porras, Clegg y Crawford, 2004).

Así, la confianza funge como mecanismo rector que logra conciliar, mediante el análisis de significados locales y la creación de nuevos significados compartidos, las culturas propias de diversos demandantes de recursos. Dado que éstos se tienen que compartir y que las estrategias originales de adquisición de los mismos se ven afectadas por el cambio de

\footnotetext{
1 Sin embargo, a pesar de que en la actualidad los miembros de las organizaciones asumen mayores responsabilidades y tareas, aun existe un clima general de incertidumbre que se traduce en desmotivación, miedo e inseguridad en el ámbito laboral.
} 
factores externos, es a partir de la confianza como se logra convertir el conflicto en colaboración.

\section{Esquema 1: La confianza como mecanismo rector para el acuerdo entre perspectivas culturales de organizaciones diferentes en demanda de recursos comunes}

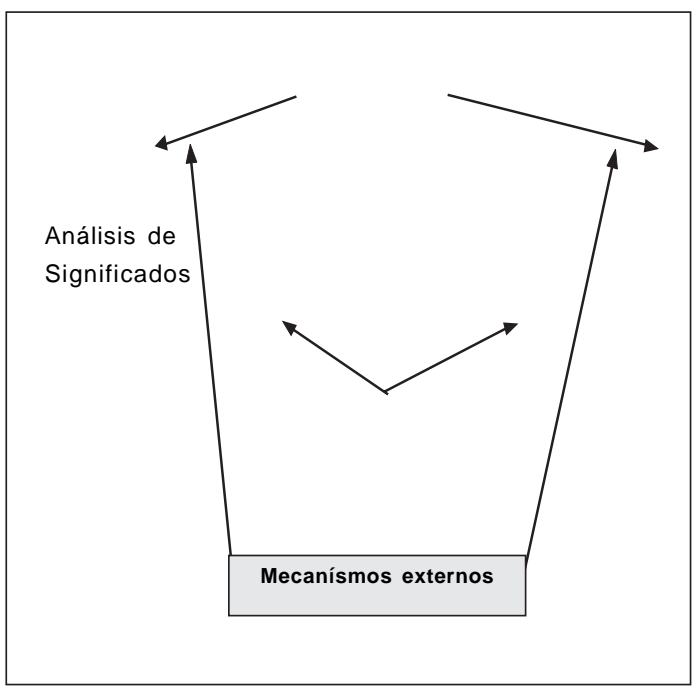

La noción de confianza ha sido llevada del ámbito social al de las organizaciones (Thuderoz et.al.,1999) mediante un discurso que permite explicar las nuevas formas en la coordinación y control del trabajo. Sin embargo, lo importante es conocer (o descubrir) cómo se genera la confianza entre organizaciones y cómo se diseñan los espacios de confianza dentro de las mismas.

Con base en la confianza se puede elaborar un acuerdo entre las organizaciones. La confianza les pone en obligación común: cada uno se somete al otro y le da crédito, se coloca y le colocan dentro de la obligación de emitir o sostener promesa (Mangematin et Thuderoz, 2003).

Las necesidades sentidas por cada una de estas organizaciones las llevan a la búsqueda de socios que las auxilien, y es así como reflexionan sobre la oportunidad de cooperación. La determinación de volver esta cooperación posible será tomada por quienes deciden una vez que han tomado opinión de los individuos de la organización. Los grupos de trabajo hacen una tarea de reflexión a fin de definir el objeto de la cooperación y sus modalidades (Mangematin et Thuderoz, 2003). En la vida actual de las organizaciones para alcanzar sus objetivos se requiere de la confianza (Mangematin y Thuderoz, 2003), y del compromiso (Lincoln y Kalleberg, 1990) como nuevos mecanismos de coordinación y de control. La confianza ha sido considerada como un elemento básico tanto para las organizaciones como para la economía en general. Al mismo tiempo la confianza es la base para lograr el compromiso de los miembros de la organización.

El fenómeno de la confianza es inherente a un contexto social específico: la comunidad (Thuderoz y Harrisson, 1999). Aunque dicho contexto no había sido considerado importante en las transacciones del mercado, recientemente, con la emergencia del concepto de redes ${ }^{1}$ y con la importancia que se le ha conferido, los investigadores y quienes diseñan las políticas se han hecho conscientes de los beneficios económicos que derivan de la colaboración basada en las relaciones de confianza en el nuevo esquema de competencia.

\footnotetext{
1 Desde los años ochenta el concepto de red ha sido utilizado en los negocios (De Bresson y Amesse, 1991; Jarillo y Ricart, 1987; Jarillo, 1988; Noria, 1992, citados por Porras, Clegg y Crawford, 2004)
} 
Entre las distintas reflexiones que suscita el tema de la confianza entre los investigadores ${ }^{6}$, sobresale aquella que devuelve la sensibilidad al ser humano para mirar hacia lo ético y actuar en consecuencia.

Así, es calificada como obligación moral, responsabilidad e interés por el otro (Braber, 1983); como lubricante necesario para el intercambio, (Shaw, 1997; Gambetta, 1988); y como proceso dinámico (Lewicki y Bunker, 1996). Se menciona la importancia de la experiencia previa y de la existencia de un capital social como bases para establecer la confianza que lleve a la formación de relaciones entre organizaciones: Larson y Starr (1993), Bidault y Jaramillo (1997), Burt (1992) y Putman (1993), Coleman (1990), Bebbington et al. (2000).

Sin lugar a dudas, una de sus características básicas para su creación y su acción es la expectativa positiva a futuro que genera. Thorelli (1986) señala que la confianza implica una relación duradera y proyecto futuro; Granovetter (1985) dice que busca un desempeño que cumpla tanto con las expectativas individuales como con las de la organización; Husted (1994) indica cómo se adquiere a largo plazo, compartiendo normas y valores; Zucker
(1986) fundamenta su doble origen: las expectativas y las reglas.

Otros autores señalan algunos elementos necesarios para establecer la confianza. Así, Bidault y Jaramillo (1997) afirman que los contratos establecen un fundamento para su acción, mientras que la ética provee las reglas y los valores que enmarcan la relación. Al mismo tiempo, Porter (1998) señala que se crea a partir de los atributos personales y de una cierta capacidad de aprendizaje.

Sin embargo, Granstrand y Sjolander (1994) advierten como la alta confianza y la colaboración pueden generar conflictos con grupos de cultura e identidad distintos, especialmente ante la falta de un gobierno de tipo jerárquico, como lo mencionan Newell y Swan (2000).

Por ejemplo, para Lewicki y Bunker (1996) la confianza se puede describir a partir de un modelo de tres niveles: 1) cálculo costobeneficio, 2) conocimiento e información, 3) identificación de los deseos e intenciones del otro. Todo esto se puede representar en el siguiente esquema:

Esquema 2: La confianza: el lubricante de las relaciones entre las rganizaciones

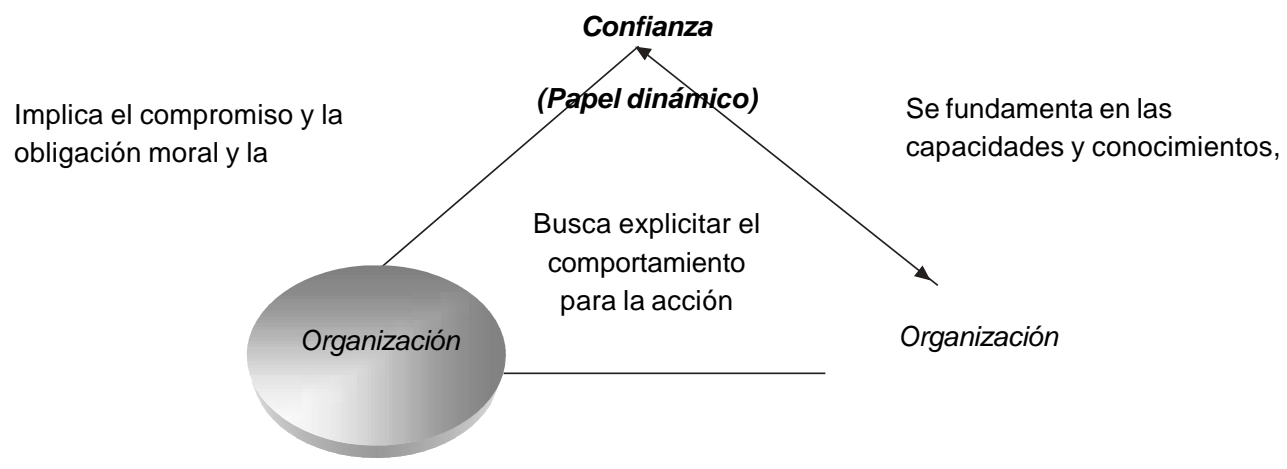

Fuente: Elaboración propia con base en Porras, Clegg y Crawford

\footnotetext{
${ }^{6}$ Porras, Clegg, Crawford (2004), hacen un recuento de las concepciones que de la confianza tienen diferentes autores.
} 
En los cuatro niveles o ámbitos de confianza: el individual, el grupal, el interorganizaciones y el intraorganización así como en el institucional, la confianza incide en la transferencia o intercambio de conocimientos, técnicas y procedimientos científicos, tecnologías, productos, capitales, entre otros. La práctica de la transferencia no es nueva, y se constituye como elemento central en las relaciones entre los diferentes países o regiones de una misma nación; relaciones en las que, aun sin ser considerada, la confianza ha estado presente.

Debido a la turbulencia y complejidad del contexto, las organizaciones y los individuos buscan, a partir de la confianza, fortalecer las relaciones laborales y sociales, para tratar de disminuir la incertidumbre que surge de las interacciones y del propio contexto.

Mangematin y Thuderoz (2003), apoyados en Gambetta y Williamson, definen la confianza como un nivel determinado de probabilidad subjetiva, estimada por un agente, respecto a lo que otro agente o un grupo se comprometerán en una acción particular. Para estos autores, la confianza se analiza desde dos perspectivas: la perspectiva racional, que se centra en las ganancias individuales con frecuencia no simbólicas, y la psicosocial, que se concentra en la identidad del grupo.

Estas perspectivas hacen evidentes dos dimensiones de la confianza: una referida a la racionalidad instrumental y al beneficio personal; otra, vinculada a las normas éticas y sociales en busca del beneficio social.

Esquema 3: Perspectivas para el análisis de la confianza

\begin{tabular}{|c|c|c|}
\hline \multicolumn{2}{|c|}{ La confianza } \\
\hline $\begin{array}{l}\text { Perspectivas } \\
\text { Enfoques }\end{array}$ & \multicolumn{1}{|c|}{ Objetivo } & \multicolumn{1}{c|}{ Teorías } \\
\hline $\begin{array}{l}\text { Racional } \\
\text { calculada }\end{array}$ & $\begin{array}{l}\text { Ganancias individuales } \\
\text { no simbólicas }\end{array}$ & $\begin{array}{l}\text { Teoría de la agencia -las relaciones } \\
\text { económicas se fundan sobre el } \\
\text { interés individual-. Teoría de juegos } \\
\text {-analiza el oportunismo de los } \\
\text { individuos-. }\end{array}$ \\
\hline Psicosocial & $\begin{array}{l}\text { Se enfoca en la } \\
\text { identidad del grupo, } \\
\text { la obediencia y la } \\
\text { implicación }\end{array}$ & $\begin{array}{l}\text { Teoría del intercambio social. Un } \\
\text { acto voluntario, que no revela interés } \\
\text { personal ni cálculo de probabilidades. }\end{array}$ \\
\hline
\end{tabular}

Fuente: Elaboración propia con base en Mangematin y Thuderoz (2003:75).

En la actualidad el mundo del trabajo enfrenta problemas importantes, entre ellos la fragmentación y la ruptura del vínculo social; por ello no es casual el énfasis de las propuestas actuales en la confianza como ese lubricante que haga funcionar las relaciones 
sociales en la organización y fuera de ella, y que permite asegurar no sólo el cumplimiento de los contratos implícitos, sino también la responsabilidad social.

Desde los niveles o ámbitos de la confianza (Mangematin y Thuderoz, 2003: 75-76) que aquí se han mencionado se pretende explicar cómo se logra establecer cierto nivel de confianza en organizaciones que por motivos de interés mutuo se vinculan, pero a la vez conservan su distinta naturaleza. Una relación de confianza en la que según explica Braber (1983, ibid.) existe una obligación moral y de responsabilidad por parte de los individuos, es decir, una dimensión ética que guía los comportamientos en función de la consecución del los logros, tanto de la organización como de los individuos.

Se puede decir que existe un proceso dinámico que provoca una transformación de la confianza, en sus diferentes niveles, en una especie de espiral en la que se adapta a las diferentes situaciones sin rupturas. Se transita de 1) la confianza calculada, que consiste en una aproximación racional económica que rinde cuentas de las transacciones de mercado, a 2) la confianza fundada sobre el conocimiento, que se refiere a la probabilidad estimada por un actor, respecto a la actuación de otro actor, estimación que no se funda en juicios de valor sino en las perspectivas de acción, y luego hacia 3) la confianza institucional, incrustada en un contexto social y organizativo, en el cual se inscriben contratos que se interesan por las relaciones entre los sistemas y los procedimientos, para controlar y coordinar los contratos y las reglas burocráticas que actúan como sustitutos de la confianza entre las personas, y que permite reducir la incertidumbre y el riesgo. Se retorna así a sus orígenes, es decir, a 4) la confianza interpersonal, fundada sobre los valores y las normas comunes.

Si la relación se nutre de una forma inicial de confianza institucional e impersonal, su logro se sostiene en la capacidad de los actores de atar una relación de confianza relacional interpersonal, que no es sino un proceso condicional, contextualizado y transicional, sin cesar sometido a las fluctuaciones de las relaciones sociales (Mangematin y Thuderoz, 2003), abierto a la dimensión ética y crítica del pensamiento (Faÿ, 2004) así como al reconocimiento del otro, a dilucidar mediante la palabra la forma como los individuos interpretan las situaciones y las reglas lógicas que aplican para decidir, al entendimiento de las posibilidades de iniciativa, la cooperación y el conflicto.

Parsons (en 1951, citado por Mangematin y Thuderoz) sugiere que la acción de crear confianza, dentro y entre las organizaciones, se puede definir como la suspensión del propio interés, en beneficio de una orientación colectiva (Mangematin y Thuderoz, 2003: 74-76). Se puede considerar como el abandono del uno hacia el otro, en el que se da crédito mutuo y conduce a situarse y ser situado en condición de obligación y compromiso, o en el don y el contra don ${ }^{1}$, para lograr una situación de equilibrio en la cual la confianza permanece activa.

\footnotetext{
${ }^{1}$ Marcel Mauss (citado por Mangematin y Thuderoz, 2003) señala que la sociedad se construye sobre un fenómeno social mayor, el don, compuesto por tres fases: dar, recibir, y después devolver. El punto de partida de esta relación se da cuando los actores dan. El don libre de carga no supone devolución definida. En este proceso el actor o los actores son libres de aceptar o de rechazar el objeto dado; pero si aceptan, pueden devolver algo a cambio para equilibrar la relación que se ha comenzado a establecer; esto es lo que el autor llama el contra don. El devolver no significa que se regresa la cosa dada, sino que se regresa otra cosa que tiene al menos el mismo valor que lo recibido. Con ello se genera un proceso dinámico en el que se da, se recibe y se devuelve, el cual permite mantener activa la confianza.
} 
La confianza en que una organización es susceptible de interrelacionarse con otra se construye por el análisis de sus discursos y sus prácticas profesionales, de sus competencias, técnicas o gerenciales; de su comportamiento como institución y de sus relaciones previas con otras instituciones, ya sean relaciones jerárquicas o transversales, entre instituciones del mismo tipo o de otro.
El momento inicial de interrelacionarse es crucial, porque es entonces cuando se establecen los primeros signos y marcas de confianza de las organizaciones; es entonces cuando entran en juego la imaginación para establecer nuevas formas de organización para el trabajo. Es en ese momento cuando se deposita en la otra organización la confianza en su intención y competencias, y se decide avalar las medidas y los medios para la aplicación del proyecto que le es propuesto.

\section{Esquema 4: Proceso dinámico de creación y reformulación de la confianza}

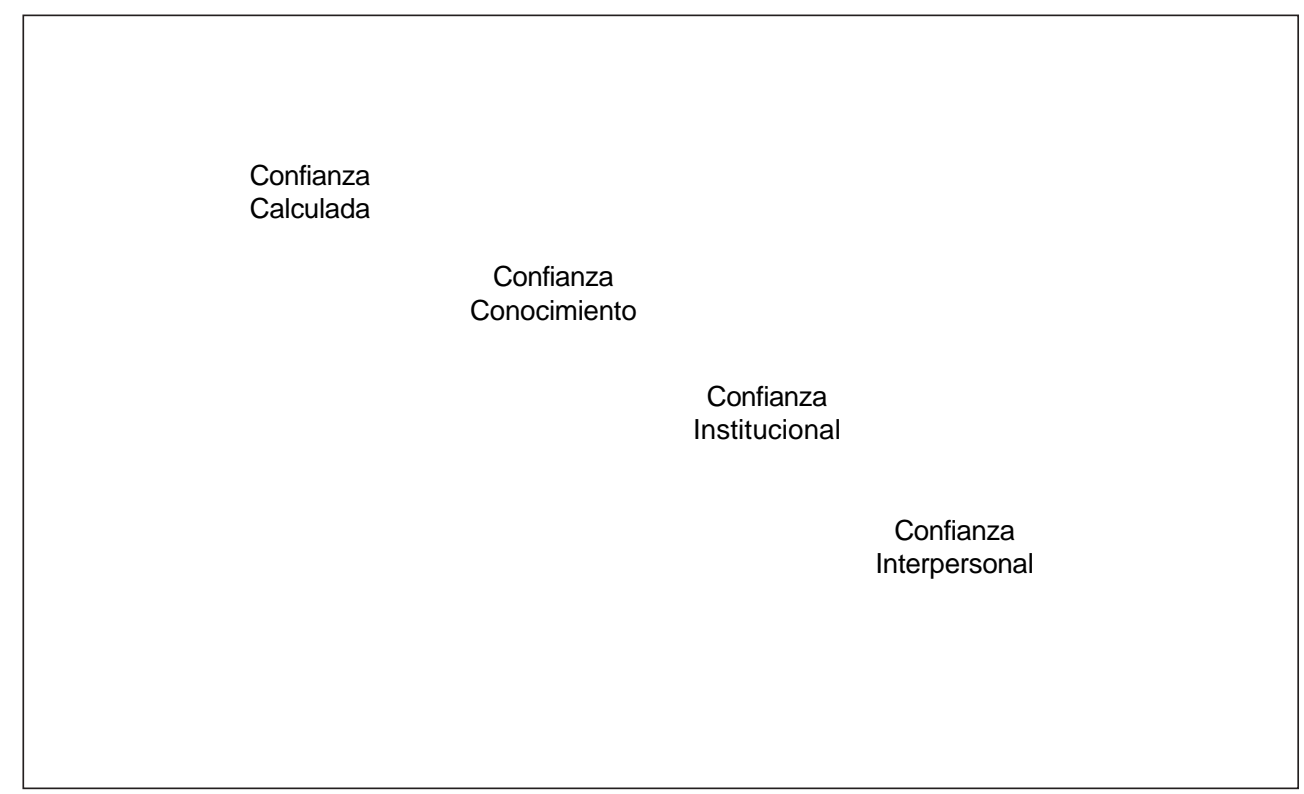

Fuente: Elaboración propia con base en Mangematin y Thuderoz (2003).

Los diferentes niveles de confianza están presentes en las relaciones que entablan los individuos, tanto dentro de la organización como en su entorno. Este entramado de relaciones con frecuencia sufre alteraciones, debido a la puesta en práctica de políticas institucionales y de la propia organización que promueven cambios técnicos y organizativos; por ello se requiere de un proceso dinámico de reformulación de la confianza a partir de lo que Rousseau (1989, citado por Mangematin y Thuderoz, 2003: 72) denomina el contrato psicológico ${ }^{1}$, definido como «la creencia individual, con respecto a los

1 El establecimiento de un contrato psicológico requiere de tomar en cuenta el contexto social y económico a nivel macro y la cultura organizacional y la práctica de los recursos humanos a nivel micro; asimismo, es importante tomar en cuenta la noción de equidad que prevalece entre los miembros de la organización, así como la confianza en los procesos de negociación en materia contractual. 
términos y las condiciones de un acuerdo de intercambio recíproco, que promete hacerse así y una compensación ofrecida en cambio».

A este proceso de constitución de la confianza no sólo contribuyen los contratos y su cumplimiento; se requiere también de creación, comunicación y compartir medios simbólicos mediante la palabra (Faÿ, 2004), la cual devuelve a los individuos la posibilidad de conocerse en su subjetividad.

\section{Esquema 5: Necesidad de la palabra para la construcción de sistemas simbólicos compartidos que hagan surgir la confianza que lleve a la acción}

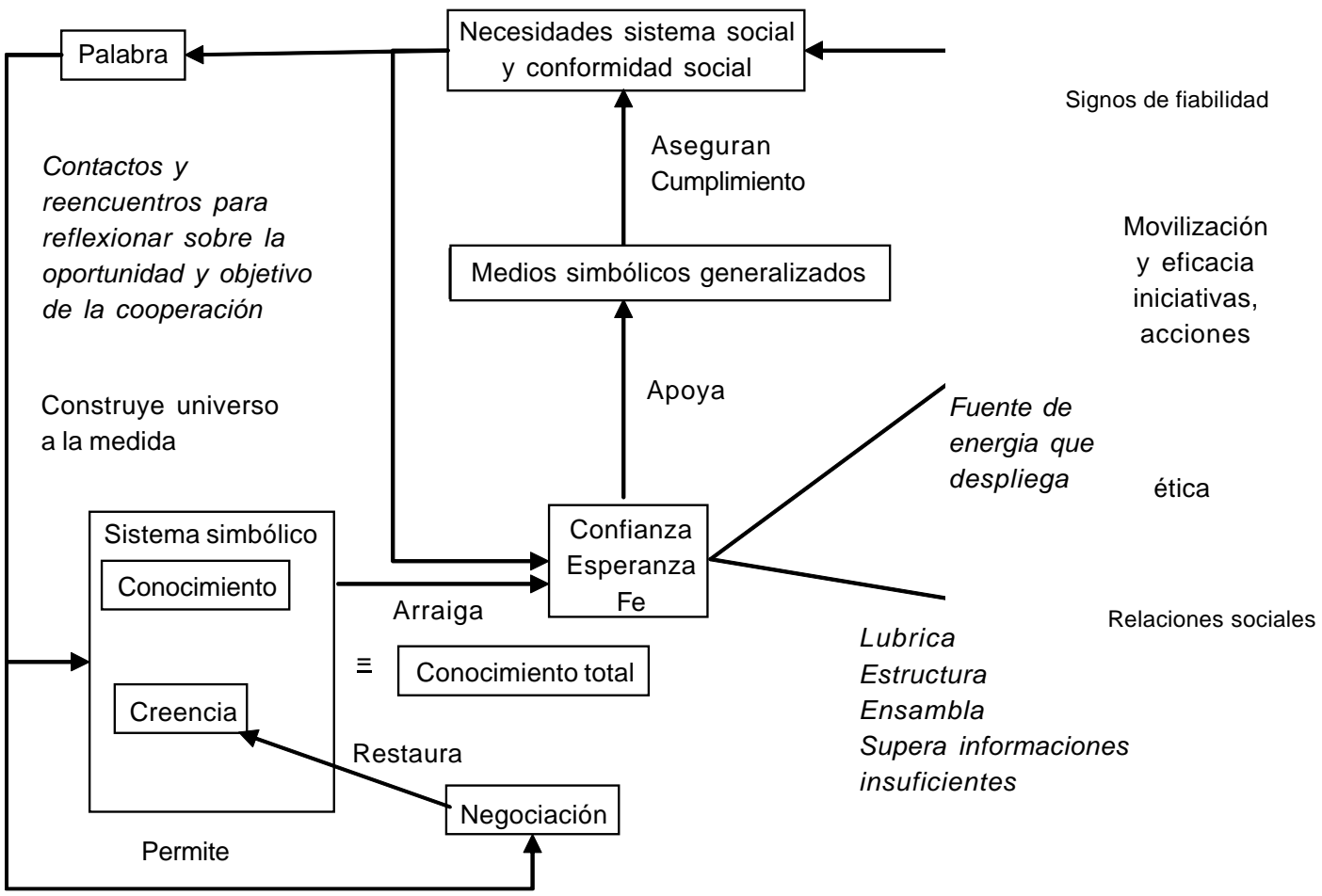

Fuente: Elaboración propia con base en Faÿ (2004), Mangematin y Thuderoz (2003). 


\section{LAS PRESIONES DEL ENTORNO HACIA LA PEQUENA EMPRESA MEXICANA}

La aplicación de los avances tecnológicos, en particular los correspondientes al campo de la informática y de la producción industrial, demanda cambios radicales en todos los ámbitos de la estructura de las organizaciones. La actuación de las empresas ha trascendido los ámbitos local y regional y ha adquirido una dimensión global. La idea de competencia entre organizaciones en el campo económico se ha transformado. Si en el pasado la competencia proponía enfrentar al competidor (en quien veía a un rival), hoy día la estrategia de supervivencia consiste en apoyar el desarrollo, buscar la cooperación y actuar globalmente mediante fusiones, adquisiciones y alianzas estratégicas con otras empresas, aun situadas en lugares distantes.

Los nuevos modelos competitivos obligan a las empresas a coexistir con culturas empresariales diferentes. Esto implica el surgimiento de procesos de pérdida de identidad, debido al desarraigo de las organizaciones. Por ejemplo, la fusión de una multinacional « $X$ » con otra "Y» $y$, a su vez, con una « $Z$ », cada una de diferente país, o bien la noción de modernización, que conduce a las organizaciones a introducir nuevas tecnologías o modernos procesos de producción en su quehacer cotidiano, afecta la identidad de las tres. ¿Qué identidad deberá adoptar cada empresa bajo las nuevas condiciones?

El constante avance tecnológico señala la preponderancia de empresas dominadas por las organizaciones que aprenden y por nuevas tecnologías de información, que carecen de hogares naturalmente determinados, de los que dispusieron en el pasado aquellas empresas que sustentaron sus ventajas comparativas en los recursos naturales y en mano de obra barata.

Sainsaulieu (1997) señala que el trabajo en la organización es fuente de afirmaciones identitarias múltiples y de contrastes. La dinámica de las organizaciones las lleva a crear nuevas identidades, especialmente cuando se introduce nueva tecnología, mediante equipos o procedimientos. Para Sainsaulieu (1997, 230-232) existen por lo menos cinco modelos de identidad:

1) el modelo reglamentario o taylorista, en el cual la confianza se deposita en las normas y reglamentos, los deberes, el status, los grados y las categorías o estratos;

2) el modelo comunitario, en el cual la confianza se funda en los afectos y fundamenta una capacidad de acción colectiva y solidaria; las manifestaciones de confianza son evidentes, sobre todo, cuando se trata de un comportamiento defensivo;

3) el modelo profesional de servicio público, que fundamenta su confianza en el saberhacer y en las competencias de los individuos que ejercen un oficio;

4) el modelo de identidad de la empresa, el cual considera que ésta es el instrumento de riesgo y de éxito colectivo e individual, por lo que, tanto los obreros como los empleados, técnicos y cuadros de mando tienen que fundamentar su confianza en la pertinencia de los objetivos de la organización y en las finalidades comunes; 
y el modelo de movilidad, en el cual la confianza se centra en la individualidad y la consecución de los proyectos personales.

Anteriormente, una empresa podía situar su casa matriz en un país y sus filiales en otras naciones y cada una tenía una identidad cultural específica. Ahora, los procesos de diseño, producción, ensambladura y comercialización de la misma empresa se pueden encontrar en diferentes puntos del mundo, y no necesariamente con filiales, y con ello se amplia el problema de la identidad.

Asimismo, el proceso de modernización por el que están pasando las PyMES mexicanas las sumerge en problemas de identidad, incluso por la puesta en práctica de nuevas formas de organización del trabajo mediante la transferencia de modelos, problemática que requiere de un redefinición de identidades.

Respecto a esto, Sainsaulieu señala que en la Francia de los años ochenta se generó, con la introducción del cambio tecnológico, un proceso de definición social llamado: identidad.

Las nuevas categorías de identidad que Sainsaulieu (1997: 218-219) descubre son: los pioneros, a quienes les atraen las innovaciones técnicas y organizacionales; los legalistas, interesados en un orden racional aceptable, es decir, que están en contra de las alteraciones del sistema social; y los excluidos, que son aquellos que no tienen ningún poder en el juego de la innovación.

En este marco de incertidumbre, hay algo claro: las nuevas condiciones contextuales impelen a las organizaciones hacia la flexibilidad y a retomar conceptos del ámbito social, como la búsqueda y la generación de confianza, para buscar su permanencia.

\section{Algunas reflexiones finales}

Es innegable la importancia de la confianza como uno de los nuevos elementos de regulación social dentro de las organizaciones; sin embargo, esta noción ha estado presente en las relaciones sociales y es hasta en los últimos años cuando ha tomado fuerza en el discurso de las organizaciones.

La confianza se convierte en un elemento de diálogo, que, mediante la palabra, permite establecer relaciones entre entidades diferentes, públicas o privadas, grandes o pequeñas, locales o extranjeras, entre otras; y probablemente fortalece sus capacidades para el logro de sus fines particulares. Sin embargo, el establecimiento de la confianza debe tomar en cuenta la prudencia, como lo sugiere Fay (2004), la prudencia o phronesis (su denominación en griego) es un modo de ejercicio de la razón que aspira al feliz éxito de la acción, una acción guiada en la confianza que tenga como resultado el bienestar para el hombre.

La pretensión de la phronesis es obtener del mejor comportamiento la verdad de la acción que conduce a la vida buena; es decir, el feliz éxito de la acción que aspira a corresponder a un bien para el hombre, bienestar que pretende ser global y durable. Con todo y la posibilidad que el hombre tiene de deliberar y de generar confianza, es evidente que entre los hombres prevalecen tanto la confianza calculada como los deseos de altruismo para entrar en la confianza interpersonal.

Si bien es cierto que el proceso de globalización ha generado la desaparición de diversas empresas y que las más vulnerables han sido las pequeñas ${ }^{1}$, también lo es que a 
partir del considerable número de éstas el país puede defenderse de la expansión comercial de las grandes potencias económicas. Además, algunas grandes empresas actualmente están promoviendo el desarrollo de encadenamientos productivos, para apoyar el desarrollo del sector industrial del país y de algunas empresas pequeñas y medianas que son sus proveedoras ${ }^{2}$; relaciones que requieren de la confianza y de la deliberación abierta, es decir, de la palabra.

\section{Bibliografía}

AKTOUF, Omar (1998). De la dirección al control, una cuestión de poder, La crítica general dirigida a la Administración clásica y el estado de crisis actual. En: La administración entre tradición y renovación. Artes Gráficas Univalle, Cali.

ALVESSON, Mats; y WILLMOTT, Hugo (2002). Identity Regulation as Organizational Control Producing The Appropriate Individual. In: Journal of Management Studies, No. 35:5, July.

CIID (2000). Cultivar la paz. Conflicto y colaboración en el manejo de los recursos naturales. BUCKLES, Daniel (ed.). Centro Internacional de Investigaciones para el Desarrollo (CIID). Ottawa, Canadá.

DE VRIES, Wieste (2001). Gobernabilidad, cambio organizacional y políticas. En: Revista de educación superior, Vol. XXX (2), No. 118 Abril-Junio, México, ANUIES.

FAÿ, Eric (2004). Information, parole et delibération. L'enterprise et la question de

l'homme Les Presses de l'Université Laval, Québec. Canadá.

HOFSTEDE, Geert (1980). Culture's Consequences. International Differences in Work-Related Values. Ed. SAGE, London.

LINCOLN, James; y KALLEBERG, Arne (1990). Culture, control and commitment. Ed. Cambridge University Press, Great Britain.

MANGEMATIN, Vincent; et THUDEROZ, Christian (2003). Des mondes de confiance. Un concept a l'épreuve de la réalité sociale. CNRS Editions, París.

SAINSAULIEU, Renaud (1997). Sociologie de l' entreprise. Organisation, Culture et Développement. $2^{\mathrm{a}}$ ed., Ed. Presses De Sciences Po et Dalloz, París.

MANGEMATIN, Thuderoz ; y HARRISSON, Denis (1999). La confiance. Approches Économiques et sociologiques. Ed. Gaetan Morin, Francia.

1 Jorge Katz (1999) menciona que, a partir del proceso de reestructuración sectorial en los países de América Latina, se ha dado paso a la muerte y nacimiento de empresas como fenómeno intrasectorial, por el cambio del peso de las ramas productivas en producto agregado de la economía.

2 Bimbo junto con Nacional Financiera, ha estado apoyando el desarrollo de encadenamientos productivos mediante el programa «Desarrollo de proveedores». 


\section{Hemerografía}

CAMARGO, Francisco Javier (2001). Management. En: El mundo ejecutivo, No. 261, Enero, Año XXII, Vol XXXVII, México, Grupo Internacional Editorial.

KATZ, Jorge (1999). Cambios en la estructura y comportamiento del aparato productivo latinoamericano en los años 1990: Después del "Consenso de Washington" ¿Qué? Conferencia para el 25‥ Aniversario de la UNAM, México, D. F.

HEYDEBRAND, Wolf V. (1989). New Organizational Forms. En: Work and Occupations, 16/3, pp. 323-357; y «Cambio en los paradigmas de la administración», en Iztapalapa, año 20, No. 48, enero-junio, Universidad Autónoma Metropolitana, México, D. F.

PORRAS, Salvador; CLEGG, Stewart; y CRAWFORD, John (2004). Trust as Networking Knowledge: Precedents from Australia. En: Asian Pacific Journal of Management, Kluwer Academic Publishers, Netherlands.
WISNER, A. (1992). De la ergonomía a la antropotecnología. La organización de la empresa y del trabajo en las transferencias de tecnología. En: Revista de Sociología del Trabajo. Empleo, trabajo y sociedad. No. 17, Invierno 92/93, Ed. Nueva Época, México.

\section{Fuentes electrónicas}

Dirección de comunicación social. Supera NAFIN en casi $50 \%$ su meta de apoyo a empresas, Nacional Financiera, Boletín de prensa No. 25/2000. México, D. F http:// www.nafin.gob.mx/noticias/29-2000.htm. ,: Venturín del Piero, Marita. «La confianza como mecanismo de regulación de las relaciones en la empresa», http://www.elzondabusiness.diarioelzonda.com. Oriol, Prats Joan, (2003), «El concepto y el análisis de la gobernabilidad", en Instituciones y desarrollo. http://www iigov.org/revista/ Salas Fumas. "Cultura y confianza en las organizaciones", de, en XXI Jornadas de la Asociación de Economía de la Salud, Oviedo, AES, España. http://www.aes.es Secretaria de Economía (1999). http:// www.economia.gob.mx. 
